\title{
Crying Without Tears: Dimensions of Crying and Relations With Ocular Dryness and Mental Well-Being in Patients With Sjögren's Syndrome
}

\author{
N. van Leeuwen ${ }^{1,2}$ - E. R. Bossema ${ }^{1}$ - R. R. Vermeer $^{1}$ A. A. Kruize ${ }^{2}$. \\ H. Bootsma ${ }^{3}$ - A. J. J. M. Vingerhoets ${ }^{4}$ J. W. J. Bijlsma ${ }^{2}$ R. Geenen ${ }^{1,2}$
}

Published online: 9 September 2015

(c) The Author(s) 2015. This article is published with open access at Springerlink.com

\begin{abstract}
This study examined dimensions of crying and its relations with ocular dryness and mental well-being in patients with Sjögren's syndrome, a systemic autoimmune disease with dryness as primary symptom. Three-hundred patients with Sjögren's syndrome completed questionnaires on crying, dryness, and well-being. The crying questionnaire revealed four dimensions: "Cryability" (comprising both crying sensibility and ability to cry), Somatic consequences, Frustration, and Suppression. Compared to 100 demographically-matched control participants from the general population, patients scored low on Cryability and high on Somatic consequences and Frustration. The crying dimensions generally showed significant but weak associations with ocular dryness and mental well-being in patients. This is the first quantitative study indicating that crying problems are more common in patients with Sjögren's syndrome than in the general population. Perhaps, patients who experience problems with crying could be helped to rely on other ways of expressing emotions than crying in tear-inducing situations.
\end{abstract}

\section{R. Geenen}

r.geenen@uu.nl

1 Department of Clinical and Health Psychology, Utrecht University, PO Box 80.140, 3508 TC Utrecht, The Netherlands

2 Department of Rheumatology and Clinical Immunology, University Medical Center Utrecht, Utrecht, The Netherlands

3 Department of Rheumatology and Clinical Immunology, University Medical Center Groningen, Groningen, The Netherlands

4 Department of Medical and Clinical Psychology, Tilburg University, Tilburg, The Netherlands
Keywords Sjögren's syndrome · Crying · Emotions · Ocular dryness

\section{Introduction}

When Henrik Sjögren introduced Sjögren's syndrome in 1933, he described 19 patients with rheumatism, dryness of the mouth, and problems with crying. Patients reported that their eyes stayed dry during crying, although they knew for sure that they were able to cry with tears before, and they also experienced irritations in the eyes, which colored red (Sjögren, 1933). In the years thereafter, Sjögren's syndrome has been firmly established as a disease, but up to now, only a few studies have addressed problems with crying in this group (Kassan, 2001; Messmer, 2009; Van Bijsterveld, Kruize, \& Bleys, 2003), and to date no research on crying has been conducted in patients with Sjögren's syndrome.

Sjögren's syndrome is a systemic autoimmune disease with a female preponderance, affecting about $0.5 \%$ of the adult population. Lymphocyte infiltration of exocrine glands results in the hallmark symptoms dryness of the eyes (keratoconjunctivitis sicca) and mouth (xerostomia; Bowman, Ibrahim, Holmes, Hamburger, \& Ainsworth, 2004; Fox, Tornwall, \& Michelson, 1999; Fox, 2005; Thomas, Hay, Hajeer, \& Silman, 1998; Vitali et al., 2002). Ocular dryness and reduced tear production may influence the ability to produce emotional tears (Kassan, 2001; Sjögren, 1933). Crying has been defined as a secretomotor phenomenon characterized by the shedding of tears from the lacrimal apparatus, without any irritation of the ocular structures, and often accompanied by alterations in the muscles of facial expression, vocalizations, and in some cases, sobbing, which is the convulsive inhaling and 
exhaling of air with spasms of the respiratory and truncal muscle groups (Patel, 1993). Crying is considered to have at least two functions. First, it is a distress signal, a powerful emotional expression that elicits attention and support from the social environment. Second, it has been attributed arousal-reduction functions that facilitate psychological and physiological recovery when being distressed (Bylsma, Vingerhoets, \& Rottenberg, 2008; Rottenberg, Bylsma, \& Vingerhoets, 2008; Vingerhoets, Cornelius, Van Heck, \& Becht, 2000).

The few articles addressing crying problems in Sjögren's syndrome documented that some patients with dry eyes report being able to produce tears after an emotional event (Van Bijsterveld et al., 2003), whereas other patients are not able to produce emotional tears, which they consider a serious problem (Kassan, 2001). This suggests that crying ability differs between individuals and that it is relevant to mental well-being. However, it is unknown whether variations in the crying ability of patients with Sjögren's syndrome reflect normal variation, whether there are multiple relevant dimensions of crying, and whether these dimensions are related to mental well-being. We expect that patients with Sjögren's syndrome on average experience more crying problems than people in the general population because of the physical limitation imposed on crying due to the dry eyes. In the general population several other aspects of crying have been distinguished such as crying sensibility, somatic and mental consequences of crying (relief, frustration), and suppression of crying (Vingerhoets, 2013). A well-known personality dimension relevant to crying is affect intensity, the strength with which emotions are experienced (Gross \& John, 1997; Larsen, Diener, \& Emmons, 1986). Affect intensity is reflected in susceptibility to crying in emotional situations, such as when saying goodbye to a beloved one or when watching a touching movie (Gross \& John, 1997; Larsen et al., 1986; Vingerhoets, 2013). Our previous study observed individual differences in affect intensity among patients with Sjögren's syndrome, but the level of affect intensity did not differ between patients and people from the general population (Van Leeuwen et al., 2012). Another aspect of crying that may be specific for the Sjögren's population involves somatic consequences, such as irritations in the eyes (Sjögren, 1933), and psychological consequences, such as frustration with crying inability (Kassan, 2001). Insight into the dimensionality of crying is important because a possible hampered ability to cry and adverse consequences of crying in patients with dry eyes may influence mental well-being and the aforementioned crying functions of signaling social distress and reducing arousal.

The aim of this study was to examine whether and which dimensions of crying can be distinguished in patients with
Sjögren's syndrome, whether these dimensions are correlated with ocular dryness and mental well-being in patients with Sjögren's syndrome, and whether differences in the scores on these crying dimensions are observed between patients with Sjögren's syndrome and a demographicallymatched comparison group from the general population. We hypothesized that several dimensions of crying problems are distinguished in patients with Sjögren's syndrome, reflecting individual differences in their ability to cry, susceptibility to crying and the somatic and psychological consequences of crying. Furthermore, we hypothesized that patients with Sjögren's syndrome experience more problems with crying than people from the general population and that crying problems are associated with ocular dryness and lower mental well-being in the Sjögren's group.

\section{Methods}

\section{Participants}

Patients with Sjögren's syndrome (SS) as primary diagnosis were included in the study. The criteria of the American-European Consensus Group on Classification criteria for Sjögren's syndrome (Vitali et al., 2002) were used. These criteria involve the occurrence of ocular and oral dryness as assessed by clinical interviews and objective tests of dryness as well as laboratory determinations of histopathology or serology (Vitali et al., 2002). Patients with Sjögren's syndrome were selected from a larger population of patients with sicca (dryness) symptoms $(N=937)$ from the Departments of Rheumatology and Clinical Immunology of the University Medical Centers (UMC) in Groningen $(n=270)$ and Utrecht $(n=667)$, The Netherlands. Patients were invited by their rheumatologist to participate in a questionnaire study. From the 937 questionnaires that were sent to patients with sicca symptoms, 470 completed questionnaires were returned (50\% response), of which 300 were from patients who fulfilled the criteria of primary Sjögren's syndrome. Respondents $(n=170)$ were excluded because they did not have the diagnosis Sjögren's syndrome $(n=21)$, because their diagnosis was non-Sjögren's sicca syndrome $(n=115)$, or because Sjögren's syndrome was not their primary diagnosis $(n=34)$. Patients who did not want to participate $(n=467)$ were asked to complete and return a "non-participation form" with questions on demographic characteristics. As compared to the patients who completed the questionnaires, the patients who filled out a non-participation form $(n=132)$ had a lower education level $\left(\chi^{2}=20.19, p<.001\right)$, and were more often single $\left(\chi^{2}=9.90, p=.04\right)$, but did not significantly differ in age, gender, or ethnicity. 
To acquire a comparison sample, Bachelor's students from the department of Clinical and Health Psychology at Utrecht University were instructed to recruit a sample comparable to the patient sample with regard to age, gender, and socioeconomic status. This control sample was recruited in the general population outside the healthcare setting. Subjects were allowed to have whatever health problem that may occur in a representative population sample, except for the diagnosis Sjögren's syndrome. From this comparison sample of 179 people, we selected one control participant to three patients using three consecutive matching criteria: gender, age, and education level (as a proxy of socioeconomic status). For example, for each three women of 60 years old with a low education level, we selected one control participant with similar characteristics, or as close as possible, starting with gender, then considering age and then education level. This yielded a demographically well-matched control sample of 100 participants. The control subjects were informed that the study was about quality of life and fatigue.

All procedures followed were in accordance with the ethical standards of the responsible committee on human experimentation (institutional and national) and with the Helsinki Declaration of 1975, as revised in 2008. Informed consent was obtained from all participants for being included in the study.

\section{Measures}

Participants filled out a questionnaire including questions on demographics and medical characteristics (disease duration, comorbidities and medication use). Hypothyroidism, lymphoma and other extraglandular manifestations as well as diseases not specifically related to Sjögren's syndrome were derived from the patient reports on comorbidities and medication use.

Medication that might influence dryness was categorized into three groups: (1) medication with moistening side effects given for other reasons than Sjögren's syndrome, e.g., diazepam, (2) medication given for other reasons than Sjögren's syndrome increasing dryness or dehydration, e.g., diuretics and omeprazole, and (3) medication being symptomatic treatment of dry eyes and mouth to prevent ophthalmological complications or to reduce problems due to reduced salivary flow and/or dental caries.

\section{Crying}

Dimensions of crying were investigated with a newly developed Sicca Crying Inventory (SCI). Expert discussion yielded the items using literature on crying and Sjögren's syndrome, existing items from crying inventories (Bylsma et al., 2008; Vingerhoets, 1995; Vingerhoets, Boelhouwer,
Van Tilburg, \& Van Heck, 2001), and in-depth interviews with patients with primary Sjögren's syndrome. The final version included 21 items to be answered on a 4-point Likert scale, with the response options " $1=$ seldom or never," " 2 = sometimes," " $3=$ regularly," and " 4 = very often." Items were about crying sensations (one item); wanting to cry with tears (two items); being able to cry with tears (five items); situational triggers of crying, such as watching certain films or saying goodbye to a loved one (four items); negative somatic consequences of crying, such as burning eyes or stiffness of the neck (four items); positive emotional consequences of crying, such as relief (one item); emotional and behavioral consequences of the inability to cry, such as acceptance, frustration, and suppression of emotions (four items). SCI items and psychometric properties are presented in Table 2 .

\section{Ocular Dryness}

The severity during the previous two days of dry eyes and of a sandy/gravel feeling in the eyes was indicated on $100 \mathrm{~mm}$ visual analogue scales between the extremes "not troublesome" (0 $\mathrm{mm})$ and "extremely troublesome" $(100 \mathrm{~mm})$. The mean score on these two scales was used as measure for ocular dryness.

\section{Mental Well-Being}

The mental component summary score (Ware, Kosinski, \& Keller, 1994) of the RAND36-item Health Survey (RAND36; VanderZee, Sanderman, Heyink, \& De Haes, 1996) was used to measure mental well-being. This score is a weighted sum of the eight subscales of this questionnaire. Two typical items are "Have you been a very nervous person?" and "Have you felt downhearted and blue?" In case of missing item scores, the individual respondent's mean score on the subscale was taken if missing items did not constitute more than half of the items. The mental component summary score has a mean of 50 and standard deviation of 10 in the general population. A higher score reflects better mental well-being.

\section{Statistical Analyses}

SPSS version 16.0 for Windows was used. A $p$ value $<.05$ was considered significant for all test statistics conducted in the study. Principal axis factoring with oblimin rotation was used to derive the dimensions of the SCI in the Sjögren's group. The number of factors was determined using the eigenvalue criterion $>1$, the scree plot of eigenvalues, and the pattern of factor loadings (Field, 2009). Criteria for excluding an item from the final version of the SCI were a factor loading $<.45$ on any single factor 
(Comrey \& Lee, 1992) or a loading $>.32$ on two or more factors (Costello \& Osborne, 2005). Scores on the SCI were computed by averaging the scores on the items belonging to a dimension. The individual respondent's mean score on the scale was taken if missing items did not constitute more than half of the items. The internal consistency of the final dimensions of the SCI was calculated using Cronbach's $\alpha$ coefficients.

Continuous data were screened for deviations from the normal score distribution. In the Sjögren's group, the skewness of the score distributions did not exceed -1.00 or 1.00 , meaning that the scores were distributed normally (e.g., Bulmer, 1979), except for the SCI dimension Suppression (1.57). In the control group, skewness of the score distributions exceeded 1.00 for the SCI dimensions Somatic consequences (2.17), Suppression (1.38) and Frustration (1.20) and for ocular dryness (1.92). To examine differences between the two groups, we used $\chi^{2}$ tests with respect to frequencies (gender, education level, marital status, work status, medication use, extraglandular manifestations, and comorbidities), the Mann-Whitney $U$-test with respect to the continuous variable with a non-normal score distribution in at least one of the two groups (the SCI dimensions Somatic consequences, Suppression, Frustration and ocular dryness), and independent samples $t$-tests for continuous variables with a normal score distribution in both groups (age, the SCI dimension Cryability, and mental well-being). To examine the magnitude of group differences on the outcome variables, Cohen's $d$ effect sizes were computed $\left[\left(M_{\text {Sjögren }}-M_{\text {Controls }}\right) / S D_{\text {Sjögren }}\right]$. Effect sizes of 0.20 , 0.50 , and 0.80 represent small, medium, and large group differences (Cohen, 1992).

Pearson's correlation coefficients, adjusted for age, gender, and medication with moistening or dryness side effects, were calculated to determine whether the crying dimensions were correlated with ocular dryness and mental well-being in the Sjögren's group. Correlation coefficients of $.10, .30$ and .50 represent small, medium, and large correlations (Cohen, 1992).

\section{Results}

\section{Participants}

Table 1 shows the demographic and medical characteristics of the patients with Sjögren's syndrome $(n=300)$ and control participants $(n=100)$. The groups did not significantly differ in age, gender, education level, marital status, and comorbidities, but the patients were more often unemployed than the control participants, more often used medication for symptomatic treatment of dry eyes and mouth, and more often had extraglandular manifestations.
For patients, the time since the first appearance of symptoms was 14.81 years $(S D=9.22)$ and the time since diagnosis was 11.21 years $(S D=8.33)$.

\section{Sicca Crying Inventory}

Factor analysis of the 21 items was performed in the patient group because the questionnaire was developed to assess crying problems in this specific group. The factor analysis yielded a Kaiser-Meyer-Olkin measure of sampling adequacy of 0.89 . This indicates that the squared partial correlations between the items are small compared to the squared correlations between the items and that the factor analysis yields distinct and reliable factors (Field, 2009). The Bartlett's test of sphericity was significant $\left(\chi^{2}=3635.67, p<.001\right)$, indicating that the null hypothesis that the variables in the correlation matrix are uncorrelated should be rejected (Field, 2009). Therefore, factor analysis was appropriate.

Table 2 shows the results of the principal axis factoring for the Sjögren's group. The scree plot of eigenvalues and the pattern of factor loadings after rotation suggested four factors, which were labeled Cryability, Somatic consequences, Frustration, and Suppression. The range of the item-factor correlations as derived from the structure matrix, was -.90 to -.64 for Cryability, .68 to .85 for Somatic consequences, .68 to .79 for Frustration, and .89 and .90 for Suppression.

Four of the 21 items were deleted; one item had only factor loadings $<.45$ and three items loaded on more than one factor $>.32$. The remaining 17 items loaded clearly on one of four factors: The first factor including two constructs was labeled "Cryability." The seven items of this factor reflect the capability to cry with tears (e.g., "I can produce tears when crying") as well as sensibility to crying (e.g., "In sad situations I can cry with tears, e.g., when saying goodbye to a loved one or when a loved one dies"). The other factors were named Somatic consequences (4 items, e.g., "I experience negative consequences of crying, such as burning eyes"), including items indicating the presence of bodily symptoms after crying, such as a headache or sore, burning eyes; Frustration (4 items, e.g., "There are situations in which I do want to cry, but I don't"), including items assessing the severity of suffering from the reduced capability to cry; and Suppression (2 items, e.g., "To avoid negative consequences of crying, I hold back from crying”), including items asking how much tears or emotions are restrained.

The psychometric properties of the final 17-item questionnaire are shown at the bottom of Table 2. The total percentage of variance explained by the four factors was $66.0 \%$. Cronbach's $\alpha$ coefficients for the four dimensions were $\geq .78$, which is considered good (Bland \& Altman, 
Table 1 Demographic and medical characteristics of patients with Sjögren's syndrome $(n=300)$ and control participants $(n=100)$

\begin{tabular}{|c|c|c|c|c|}
\hline Characteristics & Sjögren's syndrome & Control participants & Comparison & $p$ \\
\hline Age in years, mean $(S D)$ & $56.8(13.6)$ & $56.8(13.8)$ & $t=0.006$ & .99 \\
\hline \multicolumn{5}{|l|}{ Gender, $n(\%)$} \\
\hline Female & $279(93.0)$ & $91(91.0)$ & $\chi^{2}=0.002$ & .96 \\
\hline Male & $21(7.0)$ & $7(7.0)$ & & \\
\hline Unknown & & $2(2.0)$ & & \\
\hline Education level, $n(\%)^{\mathrm{a}}$ & & & $\chi^{2}=0.32$ & .85 \\
\hline Low & $73(24.5)$ & $27(27.0)$ & & \\
\hline Middle & $112(37.6)$ & $35(35.0)$ & & \\
\hline High & $113(37.9)$ & $38(38.0)$ & & \\
\hline Marital status, $n(\%)$ & & & $\chi^{2}=1.07$ & .79 \\
\hline Single & $22(7.3)$ & $5(5.0)$ & & \\
\hline With partner & $236(78.7)$ & $78(78.0)$ & & \\
\hline Divorced & $15(5.0)$ & $6(6.0)$ & & \\
\hline Widowed & $27(9.0)$ & $11(11.0)$ & & \\
\hline Work status, $n(\%) \dagger$ & & & $\chi^{2}=26.54$ & $<.001$ \\
\hline Employed $\geq 30 \mathrm{~h}$ a week & $29(9.9)$ & $21(21.0)$ & & \\
\hline Employed $<30 \mathrm{~h}$ a week & $77(26.3)$ & $40(40.0)$ & & \\
\hline Unemployed & $101(34.5)$ & $11(11.0)$ & & \\
\hline Retired & $79(27.0)$ & $25(25.0)$ & & \\
\hline Other & $7(2.4)$ & $3(3.0)$ & & \\
\hline \multicolumn{5}{|l|}{ Medication use, $n(\%)$} \\
\hline With dryness side effects & $65(21.7)$ & $19(19.0)$ & $\chi^{2}=0.32$ & .57 \\
\hline With moistening side effects & $10(3.3)$ & $1(1.0)$ & $\chi^{2}=1.53$ & .22 \\
\hline Being symptomatic treatment & $109(36.3)$ & $1(1.0)$ & $\chi^{2}=46.96$ & $<.001$ \\
\hline Extraglandular manifestations, $n(\%)$ & $100(33.3)$ & $11(11.0)$ & $\chi^{2}=18.66$ & $<.001$ \\
\hline Lymphoma & $14(4.7)$ & $0(0.0)$ & & \\
\hline Muscle and joint complaints & $37(12.3)$ & $7(7.0)$ & & \\
\hline Neuropathy & $12(4.0)$ & $1(1.0)$ & & \\
\hline Raynaud phenomenon & $12(4.0)$ & $1(1.0)$ & & \\
\hline Renal involvement & $4(1.3)$ & $0(0.0)$ & & \\
\hline SLE/Scleroderma/Dermatomyositis & $7(2.3)$ & $0(0.0)$ & & \\
\hline Thyroid involvement & $21(7.0)$ & $4(4.0)$ & & \\
\hline Other glandular symptoms & $19(6.3)$ & $0(0.0)$ & & \\
\hline Comorbidities, $n(\%)$ & $130(43.3)$ & $41(41.0)$ & $\chi^{2}=0.17$ & .68 \\
\hline Cancer & $13(4.3)$ & $3(3.0)$ & & \\
\hline Cardiovascular disease & $50(16.7)$ & $21(21.0)$ & & \\
\hline Osteoporosis & $13(4.3)$ & $3(3.0)$ & & \\
\hline Diabetes & $11(3.7)$ & $4(4.0)$ & & \\
\hline Mental problems & $27(9.0)$ & $6(6.0)$ & & \\
\hline Pulmonary diseases & $33(11.0)$ & $3(3.0)$ & & \\
\hline Other diseases & $37(12.3)$ & $14(14.0)$ & & \\
\hline
\end{tabular}

$S L E$ systemic lupus erythematosus

$\dagger$ The significance of the group difference in work status between patients and control participants was tested without the category "Other."

a Education level: Low primary school or lower vocational secondary education, Middle intermediate general secondary education or intermediate vocational education, High higher general secondary education, higher vocational education, or university education 
Table 2 Factor loadings of the original 21 items of the Sicca Crying Inventory (Instruction: Indicate for the following statements how often they occur: 'Seldom or never', 'Sometimes', 'Regularly' or
'Very often'), and Eigenvalues, percentages of explained variance, and internal consistency coefficients (Cronbach's $\alpha$ ) of the final items in patients with Sjögren's syndrome $(n=300)$

\begin{tabular}{|c|c|c|c|c|}
\hline \multirow[t]{2}{*}{ Items } & \multicolumn{4}{|c|}{ Factor loadings } \\
\hline & $\begin{array}{l}\text { Factor } \\
1\end{array}$ & $\begin{array}{l}\text { Factor } \\
2\end{array}$ & $\begin{array}{l}\text { Factor } \\
3\end{array}$ & $\begin{array}{l}\text { Factor } \\
4\end{array}$ \\
\hline \multicolumn{5}{|l|}{ Cryability } \\
\hline 2. I can produce tears when crying & -.79 & .03 & -.24 & .03 \\
\hline 4. In unpleasant, negative situations I can cry with tears (e.g. when angry or powerless) & -.84 & .12 & -.13 & .01 \\
\hline 5. In touching, positive situations I can cry with tears (e.g. when happy) & -.87 & .03 & -.13 & -.02 \\
\hline $\begin{array}{l}\text { 6. In sad situations I can cry with tears (e.g. when saying goodbye to a loved one or when a loved one } \\
\text { dies) }\end{array}$ & -.86 & .02 & -.13 & .02 \\
\hline 7. I can cry with tears when watching some films or listening to certain music & -.87 & -.02 & -.02 & -.01 \\
\hline 9. I experience positive consequences of crying, such as relief & -.65 & .17 & .06 & -.23 \\
\hline 16. If I have a 'crying feeling', my eyes become wet & -.79 & -.18 & .20 & .13 \\
\hline \multicolumn{5}{|l|}{ Somatic consequences } \\
\hline 8. I experience negative consequences of crying, such as burning eyes & .07 & .76 & .04 & .10 \\
\hline 12. My eyes are sore after crying & .03 & .86 & .00 & -.04 \\
\hline 13. My neck or shoulders are stiff after crying & -.04 & .66 & .02 & .04 \\
\hline 14. I get a headache after crying & -.12 & .72 & -.03 & .10 \\
\hline \multicolumn{5}{|l|}{ Frustration } \\
\hline 3. There are situations in which I do want to cry, but can't & .30 & .06 & .62 & .23 \\
\hline 17. If I have a 'crying feeling', it feels like I need to cry, but in the end no tears well up & .07 & .13 & .63 & .03 \\
\hline 19. I would like to be able to cry with tears & -.09 & -.05 & .79 & -.08 \\
\hline 20. I frustrates me that crying with tears is difficult for me & .26 & -.05 & .73 & .11 \\
\hline \multicolumn{5}{|l|}{ Suppression } \\
\hline 10. To avoid negative consequences of crying, I hold back from crying & .03 & .24 & -.01 & .83 \\
\hline 11. To avoid negative consequences of crying, I try to suppress my emotions & -.03 & .08 & .04 & .88 \\
\hline \multicolumn{5}{|l|}{ Deleted items ${ }^{\dagger}$} \\
\hline 1. Crying with tears is difficult for me & .39 & .17 & .54 & -.10 \\
\hline 15. I experience this crying feeling & -.42 & .06 & .69 & .01 \\
\hline 18. My illness makes it difficult for me to cry & .37 & -.01 & .65 & .13 \\
\hline 21. I have learnt to live with the fact that crying with tears is difficult for me & .31 & .10 & .44 & -.24 \\
\hline \multicolumn{5}{|l|}{ Statistics final questionnaire } \\
\hline Eigenvalue & 7.23 & 3.49 & 1.98 & 1.16 \\
\hline$\%$ explained variance & 34.4 & 16.6 & 9.4 & 5.5 \\
\hline Cronbach's $\alpha$ & .93 & .78 & .80 & .85 \\
\hline
\end{tabular}

Items with bold factor loadings were included in the factor

$\dagger$ Items deleted in the final version of the Sicca Crying Inventory because of a too low factor loading or too high cross loadings

1997). Pearson's correlation coefficients among the four dimensions varied from -.00 between cryability and suppression to -.42 between cryability and frustration. In the comparison group, Cronbach's $\alpha$ coefficients for the four dimensions were $.86, .81, .60$, and .78 , respectively.

\section{Crying}

Figure 1 displays the scores on the SCI dimensions of patients and control participants. The Cryability items were answered to occur "regularly" or "very often" by $26 \%$ of the patients and by $54 \%$ of the control participants. Somatic consequences were reported "regularly" or "very often" by $26 \%$ of the patients versus $6 \%$ of the control participants, Frustration with the reduced capability to cry by 34 versus $11 \%$, and Suppression of tears and emotions by $14 \%$ in both groups.

Table 3 shows the mean or median scores on the four SCI dimensions. Patients with Sjögren's syndrome reported significantly lower Cryability (medium effect size: $d=0.63$ ) and higher Somatic consequences (medium effect size: $d=0.69$ ) and Frustration (medium effect size: 
Fig. 1 Percentages of patients with Sjögren's syndrome (right) and control participants (left) who reported a low (seldom or never) to high (very often) frequency on the four dimensions of the Sicca Crying Inventory

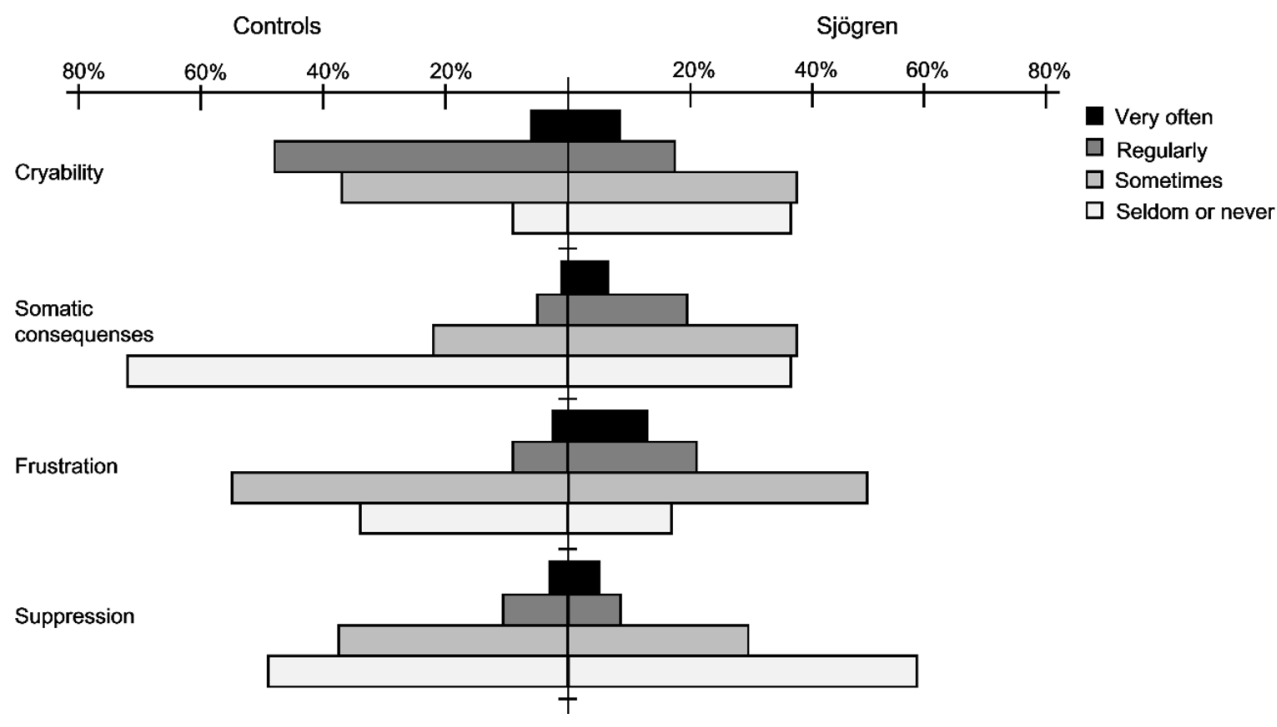

$d=0.59)$ than control participants. The two groups did not significantly differ in Suppression of tears and emotions (effect size: $d=-0.05$ ). For Cryability, we examined whether the significant difference was caused by items reflecting problems with crying ability instead of items reflecting reduced crying sensibility. This was not the case; the two groups significantly differed on all items but two: item 4 reflecting crying ability in unpleasant situations $(p=.08)$ and item 9 reflecting positive consequences of crying $(p=.11)$.

\section{Ocular Dryness and Mental Well-Being}

Table 3 also presents the median or mean scores on the measures of ocular dryness and mental well-being. Compared to control participants, patients reported more ocular dryness (large effect size: $d=1.37$ ) and lower mental well-being (small effect size: $d=0.46$ ).

Table 4 shows the partial correlation coefficients of time since first symptoms, time since diagnosis, ocular dryness and mental well-being with the four dimensions of the SCI, ocular dryness, and mental well-being in the Sjögren's group. Correlations were adjusted for age, gender, and use of medication with moistening or dryness side effects. Ocular dryness correlated negatively with Cryability (small correlation) and positively with Somatic consequences (small correlation), and Frustration (small correlation) but not with Suppression. Higher mental well-being was associated with less Somatic consequences (small correlation), less Frustration (small correlation), and less Suppression (medium correlation), but not with Cryability. Correlations of time since first symptoms and time since diagnosis with other variables were not significant.

\section{Discussion}

The current study was the first to investigate problems with crying in patients with Sjögren's syndrome and relations with ocular dryness and mental well-being. The Sicca
Table 3 Measures of central tendency, Means $(M)$ or Medians $(M d n)$, and variability, Standard Deviations $(S D)$ or Interquartile Ranges $\left(Q_{3}-Q_{1}\right)$, on the four dimensions of the Sicca Crying
Inventory (SCI), ocular dryness and mental well-being of patients with Sjögren's syndrome $(n=300)$ and control participants $(n=100)$

\begin{tabular}{|c|c|c|c|c|}
\hline & Sjögren's syndrome & Control participants & Comparison & $p$ \\
\hline \multicolumn{5}{|l|}{ SCI dimensions } \\
\hline Cryability $M,(S D)$ & $2.00(0.85)$ & $2.49(0.70)$ & $t=-5.78$ & $<.001$ \\
\hline $\begin{array}{l}\text { Somatic consequences } M d n \text {, } \\
\quad\left(Q_{3}-Q_{1}\right)\end{array}$ & $1.75(2.50-1.25)$ & $1.00(1.50-1.00)$ & $U=8127.50$ & $<.001$ \\
\hline Frustration $M d n,\left(Q_{3}-Q_{1}\right)$ & $2.00(2.75-1.50)$ & $1.75(2.00-1.25)$ & $U=9703.00$ & $<.001$ \\
\hline Suppression $M d n,\left(Q_{3}-Q_{1}\right)$ & $1.00(2.00-1.00)$ & $1.50(2.00-1.00)$ & $U=13936.50$ & .31 \\
\hline Ocular dryness (mm) Mdn, $\left(Q_{3}-Q_{1}\right)$ & $52.00(75.62-27.87)$ & $4.50(17.00-1.00)$ & $U=3846.50$ & $<.001$ \\
\hline Mental well-being $M,(S D)$ & $44.90(11.00)$ & $49.51(9.10)$ & $t=-4.15$ & $<.001$ \\
\hline
\end{tabular}


Table 4 Correlations $(r)$ of time since first symptoms, time since diagnosis, ocular dryness and mental well-being with the four dimensions of the Sicca Crying Inventory (SCI), ocular dryness and mental well-being in the Sjögren's group $(n=300)$

\begin{tabular}{|c|c|c|c|c|c|c|c|c|}
\hline & \multicolumn{2}{|c|}{ Time since first symptoms } & \multicolumn{2}{|c|}{ Time since diagnosis } & \multicolumn{2}{|c|}{ Ocular dryness } & \multicolumn{2}{|c|}{ Mental well-being } \\
\hline & $r$ & $(p)$ & $r$ & $(p)$ & $r$ & $(p)$ & $r$ & $(p)$ \\
\hline \multicolumn{9}{|l|}{ SCI dimensions } \\
\hline Cryability & .00 & $(.99)$ & .01 & $(.89)$ & -.20 & $(.001)$ & -.03 & $(.69)$ \\
\hline Somatic consequences & .04 & $(.52)$ & .03 & $(.66)$ & .27 & $(<.001)$ & -.17 & $(.005)$ \\
\hline Frustration & -.06 & $(.36)$ & .08 & $(.22)$ & .18 & $(.003)$ & -.14 & $(.03)$ \\
\hline Suppression & .02 & $(.79)$ & .03 & $(.63)$ & .09 & (.13) & -.36 & $(<.001)$ \\
\hline Ocular dryness (mm) & .01 & $(.90)$ & -.02 & $(.72)$ & & - & -.07 & $(.27)$ \\
\hline Mental well-being & .11 & $(.09)$ & -.07 & $(.25)$ & -.07 & $(.27)$ & & - \\
\hline
\end{tabular}

All correlations have been adjusted for age, gender and medication use with moistening or dryness side effects

Crying Inventory was developed for this purpose. Four crying dimensions were identified: Cryability, Somatic consequences, Frustration, and Suppression.

\section{Cryability}

Cryability involves crying sensibility as well as capability to cry. Crying sensibility appears to reflect a generic personality dimension reminiscent of affect intensity, the strength with which emotions are experienced (Gross \& John, 1997; Larsen et al., 1986). The second aspect is the actual capability of crying, which probably reflects both the constitutional ability to produce tears as well as the acquired impediment to produce tears due to Sjögren's syndrome. Although patients showed reduced Cryability (medium effect size), the large overlap between the patient and control groups on Cryability indicates that this factor only partly reflects the consequences of Sjögren's syndrome. It was expected that patients with Sjögren's syndrome might have crying problems because of the physical limitation imposed on crying due to dry eyes, but we did not expect a deviation from the general population on the sensibility aspect of crying. However, additional analysis showed that it was not exclusively crying ability that caused the difference between patients and the general population sample; both the items reflecting crying ability and the items reflecting crying sensibility explained the low Cryability scores in patients with Sjögren's syndrome. This might suggest that lower crying ability not (only) is a specific consequence of dryness, but (also) a more generic consequence of having a chronic disease.

Crying has hardly been examined in chronic diseases (Vingerhoets \& Scheirs, 2001). Preliminary research, involving poorly defined patient groups, indicated that-as compared to healthy controls-ulcer patients and colitis patients perhaps cry less frequently and evaluate crying more negatively than healthy controls (Crepau, 1981). Another study observed that patients with rheumatoid arthritis who were able to cry with tears in an emotional experiment had better disease control than patients who were not able to cry with tears (Ishii, Nagashima, Tanno, Nakajima, \& Yoshino, 2003). A reduction of crying ability may be determined by a multitude of factors independent of ocular dryness that may differ between people with and without a chronic disease such as emotional (e.g., depressive mood), cognitive and social context variables (Bylsma et al., 2008; Messmer, 2009; Rottenberg, Bylsma, Wolvin, \& Vingerhoets, 2008; Vingerhoets, Van den Berg, Kortekaas, Van Heck, \& Croon, 1993; Vingerhoets et al., 2000; Vingerhoets et al., 2001; Vingerhoets, 2013).

In the present study, the association between Cryability and ocular dryness was low, and between Cryability and mental well-being even absent. Whereas we previously observed that higher affect intensity was associated with lower mental well-being in patients with Sjögren's syndrome (Van Leeuwen et al., 2012), the current absence of a correlation between Cryability and mental well-being indicates that low crying ability and sensibility are not a serious problem in patients with Sjögren's syndrome. It could be hypothesized that the impact of ocular dryness on the ability to cry depends on the stage of the disease, for example, that first the production of basal tears stops, then the reflex tears, and finally the emotional tears (Messmer, 2009). However, we did not find a significant relation between ocular dryness and time since the first symptoms and time since diagnosis, despite the heterogeneity of the patient group on both characteristics. Thus, although Cryability is a factor that differentiates individuals within the patient group and groups of patients and controls, there is no indication that reduced crying ability and sensibility 
are of relevance for well-being in patients with Sjögren's syndrome.

Overall, the present study demonstrates that ocular dryness is not a condition sine qua non for crying ability. Rather, patients with Sjögren's syndrome are more at risk of having a reduced ability to cry with tears.

\section{Somatic Consequences}

The patient group showed more somatic consequences of crying than the comparison group (medium effect size). Somatic consequences of crying showed a medium association with ocular dryness and a small, negative association with mental well-being. Adverse somatic consequences may hamper the reduction of physiological arousal that is considered a core function of crying when being distressed (Bylsma et al., 2008; Rottenberg et al., 2008; Vingerhoets et al., 2000). In the general population, headaches and facial discomfort are frequently reported symptoms after crying (Blau, 1995). In a large online study concerning crying in 35 countries, about $7 \%$ reported stopping crying to prevent physical effects (Bylsma et al., 2008). Patients with Sjögren's syndrome can try to overcome the adverse somatic consequences of crying by using tear drops to reduce or prevent burning eyes, or perhaps by relaxation therapy to prevent or reduce a stiff neck or shoulders.

\section{Frustration}

Patients experienced more frustration because of the reduced ability to cry than the control participants (medium effect size). Correlations of frustration with ocular dryness and reduced mental well-being were small. Patients who are frustrated about their crying inability may need other ways to express their feelings and to elicit emotional support (Hendriks, Croon, \& Vingerhoets, 2008; Vingerhoets et al., 2000). Our previous study showed that processing and regulating emotions in patients with Sjögren's syndrome generally does not deviate from normal (Van Leeuwen et al., 2012). This may imply that in some patients' frustration due to the reduced ability to cry can be compensated by intact alternative ways of regulating emotions, such as expression with words or facial expression.

\section{Suppression}

No differences between the Sjögren's group and the comparison group were found with respect to the suppression of emotions or tears, the fourth SCI dimension. Patients do not report holding back their tears and emotions more often than people from the general population. This dimension showed a small negative association with mental well-being. It has been suggested that patients suffering from other chronic diseases, such as asthma (Miller, 1987; Miller \& Wood, 1997) or migraine (Evans, 1998), sometimes refrain from crying in order to prevent an attack, or to prevent further exacerbation. In general, suppression of emotions has been found to have negative consequences for affect and well-being (Gross \& John, 2003). With respect to crying, weak connections between the suppression of crying and mood improvement after crying have been observed (Bylsma et al., 2008). Suppression of tears has sometimes been thought to endanger physical health, leading to headaches, ulcers, hypertension, and insomnia (Cornelius, 1986; Vingerhoets, 2013), but empirical evidence in support of this hypothesis is largely lacking. Although low Cryability, somatic symptoms after crying, and frustration with crying are more prevalent among patients with Sjögren's syndrome than in the general population, this apparently does not lead to holding back tears and emotions.

\section{Strengths and Limitations}

This was the first study that, inspired by clinical observations by Henrik Sjögren (1933), examined problems with crying in patients with Sjögren's syndrome. The findings of the current study suggest some relevance of crying problems for mental well-being. The large sample size of patients with Sjögren's syndrome diagnosed by a rheumatologist and the large sample size of the comparison group demographically well-matched to the patient group are major strengths of our study. However, there are also limitations. First, the descriptive and cross-sectional nature of the study prevents causal inferences. Second, we did not have the option to include a comparison group with a comparable burden of disease but without obstructed tear production. Third, recruitment of the comparison sample may have caused an unforeseen selection bias; however, care was taken that the control group was well-matched to the patient group with respect to several characteristics. Fourth, we did not use observations of ocular dryness in addition to the self-report measures. Finally, we developed a crying questionnaire because it did not yet exist; the current study addresses the validity of this questionnaire preliminarily. Future research should aim at cross-validating the scales and replicating the group comparison in another sample. 


\section{Conclusion}

Although crying problems are not exclusive for patients with ocular dryness, but occur in the general population as well, patients with Sjögren's syndrome report less crying sensibility and ability and more somatic consequences and frustration with crying than people from the general population. These crying problems are associated to a small extent with more ocular dryness and reduced mental wellbeing. Perhaps patients who experience problems with crying could be helped to rely on other ways of expressing emotions than crying in tear-inducing situations.

Acknowledgments This work was financially supported by the Dutch Arthritis Association. We thank the patient representatives of the Utrecht Psychorheumatology Research Unit for their help.

\section{Compliance with Ethical Standards}

Conflict of interest N. van Leeuwen, E.R. Bossema, R.R. Vermeer, A. A. Kruize, H. Bootsma, A.J.J.M. Vingerhoets, J.W.J. Bijlsma, and R. Geenen declare that they have no conflict of interest.

Human and Animal Rights and Informed Consent All procedures followed were in accordance with the ethical standards of the responsible committee on human experimentation of the Medical Ethical Committee of University Medical Center Utrecht and with the Helsinki Declaration of 1975, as revised in 2008. Informed consent was obtained from all patients for being included in the study.

Open Access This article is distributed under the terms of the Creative Commons Attribution 4.0 International License (http://crea tivecommons.org/licenses/by/4.0/), which permits unrestricted use, distribution, and reproduction in any medium, provided you give appropriate credit to the original author(s) and the source, provide a link to the Creative Commons license, and indicate if changes were made.

\section{References}

Bland, J. M., \& Altman, D. G. (1997). Cronbach's alpha. British Medical Journal, 314(7080), 572.

Blau, J. (1995). Crying headache. Lancet, 345, 803.

Bowman, S. J., Ibrahim, G. H., Holmes, G., Hamburger, J., \& Ainsworth, J. R. (2004). Estimating the prevalence among Caucasian women of primary Sjögren's syndrome in two general practices in Birmingham, UK. Scandinavian Journal of Rheumatology, 33, 39-43.

Bulmer, M. G. (1979). Principles of statistics. New York: Dover.

Bylsma, L. M., Vingerhoets, A. J. J. M., \& Rottenberg, J. (2008). When is crying cathartic? an international study. Journal of Social and Clinical Psychology, 27, 1165-1187.

Cohen, J. (1992). A power primer. Psychological Bulletin, 112, $155-159$.

Comrey, A. L., \& Lee, H. B. (1992). A first course in factor analysis (2nd ed.). Hilsdale: Lawrence Erlbaum Associates Inc.

Cornelius, R. R. (1986). Prescience in the pre-scientific study of weeping? A history of weeping in the popular press from the mid-1800s to the present. Paper presented at the 57th annual meeting of the Eastern Psychological Association, New York $(\mathrm{NY})$.
Costello, A. B., \& Osborne, J. W. (2005). Best practices in exploratory factor analysis: Four recommendations for getting the most from your analysis. Practical Assessment, Research \& Evaluation, 10, 1-9.

Crepau, M. T. (1981). A comparison of the behavior patterns and meanings of weeping among adult men and women across three health conditions. Dissertation Abstracts International, 42, 137B-138B.

Evans, R. W. (1998). Crying migraine. Headache, 38, 799-800.

Field, A. (2009). Exploratory factor analysis. Discovering statistics using SPSS (3rd ed., pp. 627-685). London: SAGE Publications Ltd.

Fox, R. I. (2005). Sjögren's syndrome. Lancet, 366, 321-331.

Fox, R. I., Tornwall, J., \& Michelson, P. (1999). Current issues in the diagnosis and treatment of Sjogren's syndrome. Current Opinion in Rheumatology, 11, 364-371.

Gross, J. J., \& John, O. P. (1997). Revealing feelings: Facets of emotional expressivity in self-reports, peer ratings, and behavior. Journal of Personality and Social Psychology, 72, 435-448.

Gross, J. J., \& John, O. P. (2003). Individual differences in two emotion regulation processes: Implications for affect, relationships, and well-being. Journal of Personality and Social Psychology, 85, 348-362.

Hendriks, M. C. P., Croon, M. A., \& Vingerhoets, A. J. J. M. (2008). Social reactions to adult crying: The help-soliciting function of tears. Journal of Social Psychology, 148, 22-41.

Ishii, H., Nagashima, M., Tanno, M., Nakajima, A., \& Yoshino, S. (2003). Does being easily moved to tears as a response to psychological stress reflect response to treatment and the general prognosis in patients with rheumatoid arthritis? Clinical and Experimental Rheumatology, 21, 611-616.

Kassan, S. S. (2001). Managing dry eyes and dry mouth in Sjögren's syndrome. The American Journal of Managed Care, 7, S444S450.

Larsen, R. J., Diener, E., \& Emmons, R. A. (1986). Affect intensity and reactions to daily life events. Journal of Personality and Social Psychology, 51, 803-814.

Messmer, E. M. (2009). Emotional tears. [Emotionale Tränen]. Ophthalmologe, 106, 593-602.

Miller, B. D. (1987). Depression and asthma: A potentially lethal mixture. Journal of Allergy and Clinical Immunology, 80, 481-486.

Miller, B. D., \& Wood, B. L. (1997). Influence of specific emotional states on autonomic reactivity and pulmonary function in asthmatic children. Journal of the American Academy of Child and Adolescent Psychiatry, 36, 669-677.

Patel, V. (1993). Crying behavior and psychiatric disorder in adults: A review. Comprehensive Psychiatry, 34, 206-211.

Rottenberg, J., Bylsma, L. M., \& Vingerhoets, A. J. J. M. (2008a). Is crying beneficial? Current Directions in Psychological Science, 17, 400-404.

Rottenberg, J., Bylsma, L. M., Wolvin, V., \& Vingerhoets, A. J. J. M. (2008b). Tears of sorrow, tears of joy: An individual differences approach to crying in Dutch females. Personality and Individual Differences, 45, 367-372.

Sjögren, H. (1933). Zur kenntnis der keratoconjunctivitis sicca: Keratitis filiformis bei hypofunktion der tränendrüsen [On knowledge of keratoconjunctivitis sicca. Keratitis filiformis due to lacrimal gland hypofunction]. Acta Ophthalmologica Supplementum, 2, 1-151.

Thomas, E., Hay, E. M., Hajeer, A., \& Silman, A. J. (1998). Sjögren's syndrome: A community-based study of prevalence and impact. British Journal of Rheumatology, 37, 1069-1076.

Van Bijsterveld, O. P., Kruize, A. A., \& Bleys, R. L. A. W. (2003). Central nervous system mechanisms in Sjögren's syndrome. British Journal of Ophthalmology, 87, 128-130. 
Van Leeuwen, N., Bossema, E. R., Van Middendorp, H., Kruize, A., Bootsma, H., Bijlsma, J. W. J., \& Geenen, R. (2012). Dealing with emotions when the ability to cry is hampered: Emotion processing and regulation in patients with primary Sjögren's syndrome. Clinical and Experimental Rheumatology, 30, 492-498.

VanderZee, K. I., Sanderman, R., Heyink, J. W., \& De Haes, H. (1996). Psychometric qualities of the RAND 36-item health survey 1.0: A multidimensional measure of general health status. International Journal of Behavioral Medicine, 3, 104-122.

Vingerhoets, A. J. J. M. (1995). Questionnaire on adult crying. Tilburg: Tilburg University Press.

Vingerhoets, A. J. J. M. (2013). Why only humans weep. Unravelling the mysteries of tears. Oxford: Oxford University Press.

Vingerhoets, A. J. J. M., Boelhouwer, A. J. W., Van Tilburg, M. A. L., \& Van Heck, G. L. (2001). The situational and emotional context of adult crying. In A. J. J. M. Vingerhoets \& R. R. Cornelius (Eds.), Adult crying: A biopsychosocial approach (pp. 71-89). Hove: Brunner-Routledge.
Vingerhoets, A. J. J. M., Cornelius, R. R., Van Heck, G. L., \& Becht, M. C. (2000). Adult crying: A model and review of the literature. Review of General Psychology, 4, 354-377.

Vingerhoets, A. J. J. M., \& Scheirs, J. G. M. (2001). Crying and health. In A. J. J. M. Vingerhoets \& R. R. Cornelius (Eds.), Adult crying. A biopsychosocial approach (pp. 227-246). BrunnerRoutledge: Hove.

Vingerhoets, A. J. J. M., Van den Berg, M. P., Kortekaas, R. T. J., Van Heck, G. L., \& Croon, M. A. (1993). Weeping: Associations with personality, coping, and subjective health status. Personality and Individual Differences, 14, 185-190.

Vitali, C., Bombardieri, S., Jonsson, R., Moutsopoulos, H. M., Alexander, E. L., Carsons, S. E., ... Weisman, M. H. (2002). Classification criteria for Sjögren's syndrome: A revised version of the European criteria proposed by the American-European consensus group. Annals of the Rheumatic Diseases, 61, 554-558.

Ware, J. E., Kosinski, M., \& Keller, S. D. (1994). SF-36 physical and mental health summary scales: A user's manual. Boston: The Health Institute. 